\title{
Experimental assessment of photonic integrated switches based optical data center networks with virtual network slice services
}

\author{
Citation for published version (APA): \\ Xue, X., Prifti, K., Wang, F., Yan, F., Pan, B., Guo, X., \& Calabretta, N. (2019). Experimental assessment of \\ photonic integrated switches based optical data center networks with virtual network slice services. In 2019 18th \\ International Conference on Optical Communications and Networks, ICOCN 2019 [8934002] Institute of \\ Electrical and Electronics Engineers. https://doi.org/10.1109//COCN.2019.8934002
}

DOI:

10.1109/ICOCN.2019.8934002

Document status and date:

Published: 01/08/2019

\section{Document Version:}

Accepted manuscript including changes made at the peer-review stage

\section{Please check the document version of this publication:}

- A submitted manuscript is the version of the article upon submission and before peer-review. There can be important differences between the submitted version and the official published version of record. People interested in the research are advised to contact the author for the final version of the publication, or visit the DOI to the publisher's website.

- The final author version and the galley proof are versions of the publication after peer review.

- The final published version features the final layout of the paper including the volume, issue and page numbers.

Link to publication

\footnotetext{
General rights

- You may freely distribute the URL identifying the publication in the public portal. follow below link for the End User Agreement:

www.tue.nl/taverne

\section{Take down policy}

If you believe that this document breaches copyright please contact us at:

openaccess@tue.nl

providing details and we will investigate your claim.
}

Copyright and moral rights for the publications made accessible in the public portal are retained by the authors and/or other copyright owners and it is a condition of accessing publications that users recognise and abide by the legal requirements associated with these rights.

- Users may download and print one copy of any publication from the public portal for the purpose of private study or research.

- You may not further distribute the material or use it for any profit-making activity or commercial gain

If the publication is distributed under the terms of Article $25 \mathrm{fa}$ of the Dutch Copyright Act, indicated by the "Taverne" license above, please 


\title{
Experimental Assessment of Photonic Integrated Switches Based Optical Data Center Networks with Virtual Network Slice Services
}

\author{
Xuwei Xue*, Kristif Prifti, Fu Wang, Fulong Yan, Bitao Pan, Xiaotao Guo, Nicola Calabretta \\ IPI-ECO Research Institute, Eindhoven University of Technology, Eindhoven, the Netherlands \\ e-mail: x.xue.1@tue
}

\begin{abstract}
An SDN-controlled DCN based on photonic integrated switches is experimentally assessed. Network-slice can be reconfigured within $150 \mathrm{~ms}$ and deployed photonic integrated switches perform error-free $10 \mathrm{~Gb} / \mathrm{s}$ switching with $<2 d B$ penalty and zero packet-loss.
\end{abstract}

Keywords: Optical data center, Virtual network, Photonic integrated switches

\section{INTRODUCTION}

With the emerging of cloud computing, Internet of Things, and the 5G mobile communications, the traffic communications inside the data centers (DC) are imposed stringent requirements in terms of low latency, high capacity, and high cost and power-efficiency [1]. With the aim to satisfy the scalable growth in both network traffic volume and connected endpoints while decreasing the cost and the energy consumption, transparent optical DC networks (DCNs) based on fast optical switches have been considered, featuring the data rate and format transparency and eliminating the power consuming $\mathrm{O} / \mathrm{E} / \mathrm{O}$ conversions [2,3].

Commercial discrete components have been deployed in developed prototypes to implement the fast optical switch [4], whereas the practical implementation would require the integration of hundreds or even more of those optical components, resulting in power-inefficient bulky systems. The realization of photonic integrated circuits brings light to this, with the promises of reduced footprint and power consumption [5,6]. It would be especially beneficial for application scenarios like the DCNs, resulting in diminished networking complexity, easy management and less concerns with the cooling issues.

Despite these advantages of photonic integrated switches based DCN, a flexible and reconfigurable network infrastructure that enables the full exploitation of the high network throughput and nanoseconds reconfiguration time is essential. Moreover, with the critical pressure in terms of capacity and interconnectivity from the emerging traffic-boost applications, future DCNs require to allow the deployment of multiple virtual network slices (NS) over the same physical infrastructure to achieve efficient resource utilization with high tenant density [7].

In this work, we propose and experimentally assess a software-defined network (SDN) enabled virtual optical DCN based on nanoseconds WDM Photonic integrated switches (PISs). Where more than 100 components including the SOAs, AWGs and couplers are integrated in the $4 \times 4$ WDM nanoseconds PIS. An Orchestration plane and an SDN controller is deployed to enable the dynamic provisioning and reconfiguration of virtual NS. Upon the decision of the Orchestrator based on the monitored network statistics (counts of lost and retransmitted packets), the SDN controller then re-configures the path to reduce the packet loss achieving a guaranteed quality of service (QoS).

\section{SDN-ENABLED DCN BASED ON PHOTONIC INTEGRATED SWITCHES}

The optical DCN with distributed Photonic integrated switches and flow control empowered by SDN control plane is depicted in Fig. 1(a). The $\mathrm{N} M \times \mathrm{M}$ intra-cluster Photonic integrated switches (ISes) and $\mathrm{M} \mathrm{N} \times \mathrm{N}$ intercluster Photonic integrated switches (ESes) are dedicated

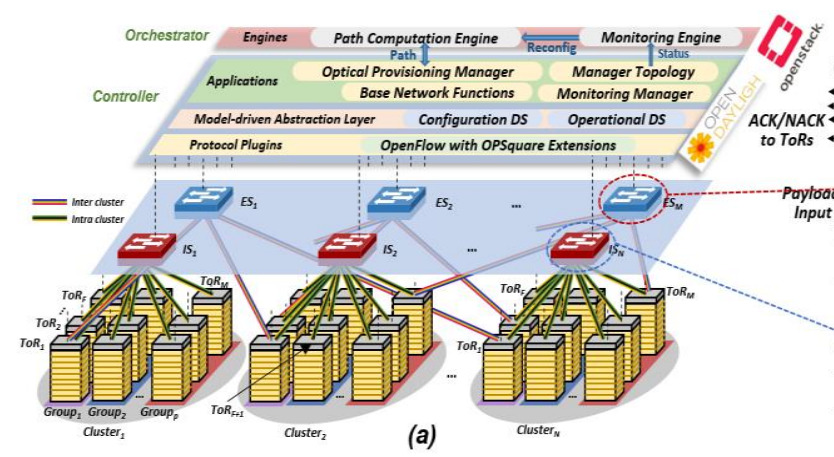

Figure. 1: (a) SDN-enabled virtual optical DCN employing Photonic integrated switches; (b) Schematic of the Photonic integrated switch; (c) Fabricated Photonic integrated switch chip. 
for intra-cluster and inter-cluster communication, respectively. The optical flow control between ToRs and IS/ES is implemented to solve possible contention. The flow control signals (ACK/NACK) are generated by the IS/ES controller and sent back to the top of rack (ToR) for releasing the packets or requesting a packet retransmission. FPGAs are used to implement the ToRs, IS/ES switch controllers and the flow control protocol.

The OpenDaylight (ODL) platform is set as the base SDN controller connecting the IS/ESes and ToRs by means of integrated OpenFlow (OF) agents implementing an extended OF protocol. The data plane layout and physical distribution information is stored in the Topology Manager (TM). The Optical Provisioning Manager (OPM) module is developed to configure the underlying devices (i.e., IS, ES and ToR) required to set up the specific network connectivity for NS deployment. The OPM contacts the Path Computation Engine (PCE) of the OpenStack-based orchestrator, which relies on abstracted topological information from the TM to provide the ODL controller with a ToR-to-ToR path computation service that computes the best NS deployment (placement and interconnection). Furthermore, the Monitoring Manager (MM) collects optical data plane statistics and aggregates them into an NS level. Such aggregated information is collected by the Monitoring Engine (ME) of the orchestrator to trigger the needed actions (e.g. NS reconfiguration) to maintain the expected QoS.

The buffer-less Photonic integrated switch is schematically shown in Fig. 1(b). The arrayed waveguide gratings (AWG) groups WDM wavelengths coming from different ToRs and each respective optical module consists of a 1: $\mathrm{N}$ splitter to broadcast the WDM channels to the $\mathrm{N}$ wavelength selective switches (WSS). The outputs of the N WSSs are connected to the respective N output ports. Each WSS can select one wavelength channel and forward the channel to the output port according to the switching control signals. Turning on/off the N SOAs determines which wavelength channel is forwarded to the output or is blocked. As shown in Fig.

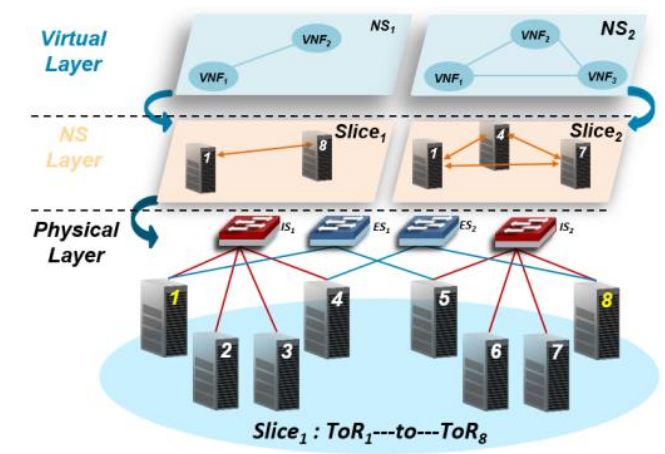

Figure. 2: Experimental set-up

1(b) each optical module forwards the input WDM signals from the ToRs in the cluster by the WSSs to the other ToRs residing in the cluster. Specifically, the $6 \times 4 \mathrm{~mm} 2$ fabricated photonic switch chip in Fig. 1(c) integrates 4 optical modules that can been used to implement 4 ES or 4 IS for 4 ToRs intra-cluster or inter-cluster interconnection. At the input of each module, an $800 \mu \mathrm{m}$ booster SOA is employed to compensate the $6 \mathrm{~dB}$ losses of the 1:4 splitter and partially the AWGs losses at the WSS. The passive 1:4 splitter is realized by cascading $1 \times 2$ multimode interferometer (MMI). Each of the four identical modules processes one of the four WDM inputs and forwards them to the dedicated outputs. Therefore, one Photonic integrated switch chips can be used to implement the 2 ES and 2 IS switches to interconnect 8 ToRs grouped in 2 clusters.

\section{EXPERIMENTAL SET-UP AND RESULTS}

The experimental set-up to assess the SDN-enabled virtual optical DCN based on the nanoseconds Photonic integrated switches is shown in physical layer of Fig. 2. It consists of 8 FPGA-based ToRs grouped in 2 clusters, connected by 2 ES and 2 IS equipped FPGA-based controller and OF agents. An ODL-based controller is placed over the data plane to control the configuration and updating of the network interconnectivity. The
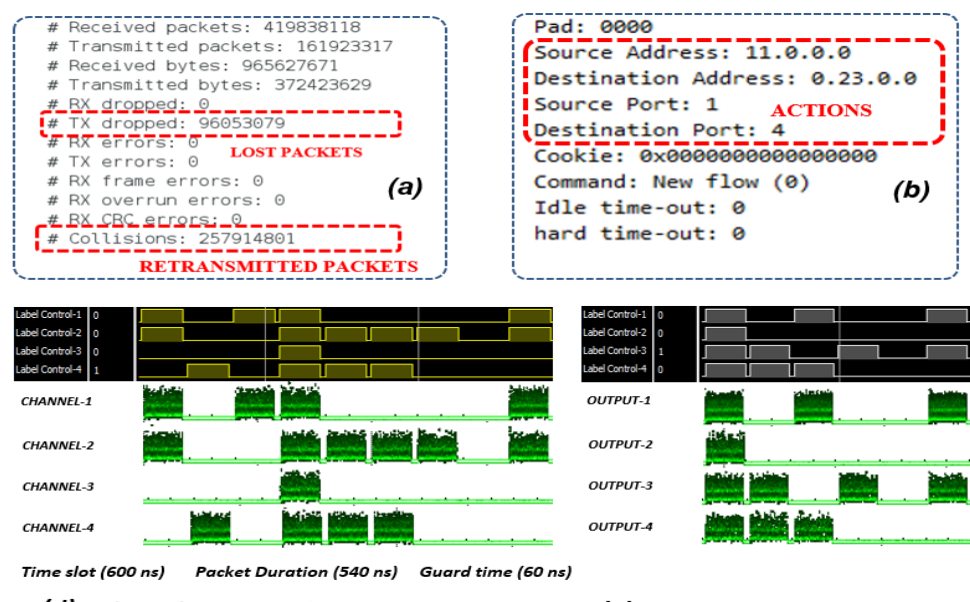

(d) 4 Channels in WSS-1 $\rightarrow$ OUTPUT-1

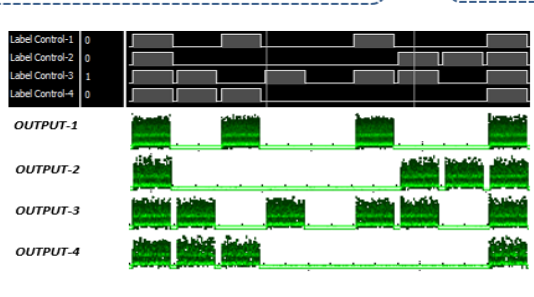

(e) Channel-1 in WSS-1 $\rightarrow 4$ OUTPUTs
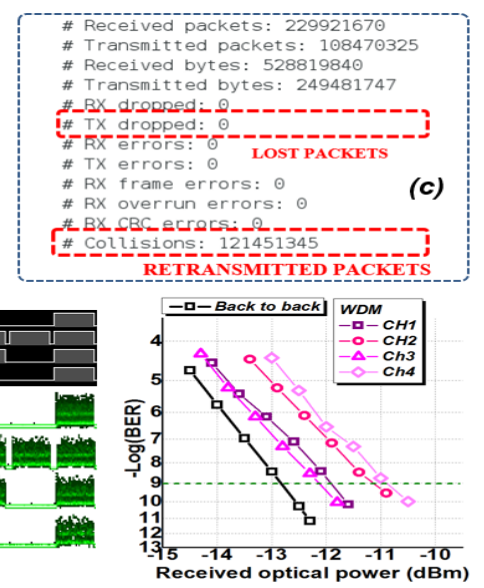

(f) BER versus receive optical power

Figure. 3: (a) Statistics monitoring before NS reconfiguration; (b) OF FlowStats used to update the set of actions; (c) Statistics monitoring after NS reconfiguration; (d) Traces for WSS1; (e) Traces for 4 outputs; (f) BER for WDM channels. 
Orchestrator implements the management operations. The first and second modules of PIS are used to implement the intra-cluster switches IS-1, IS-2 that interconnects the 4 ToRs of cluster 1 , cluster 2 , respectively. The third and fourth modules of PIS are utilized to implement the intercluster switches ES-1, ES-2 that interconnects the $2 \mathrm{i}$-th ToRs of these 2 clusters ( $i=1,4)$. The FPGA-based ToRs of cluster 1 is equipped with $10 \mathrm{~Gb} / \mathrm{s}$ SFP transceivers at $1525.0 \mathrm{~nm}, 1528.9 \mathrm{~nm}, 1532.9 \mathrm{~nm}$ and $1536.8 \mathrm{~nm}$ to generate the distinct optical packets for the four ToRs, respectively. The optical packets time slot is $600 \mathrm{~ns}$ (540 ns payload time and $60 \mathrm{~ns}$ guard time).

First, the provisioning and reconfiguration of NS infrastructure are investigated to validate the configurability of optical DCN. Each NS comprises several virtual network function (VNF), and interconnections among them that can be flexibly reconfigured depending on physical resource distribution and availability. Fig. 2 shows an example of NS allocation, where NS1 consists of VNF1 and VNF2. First, the PCE allocates VNF1 and VNF2 in racks 1 and 8 respectively according to the network resource availability. Next, the OPM coordinates with the PCE to configure the best path among ToR1 and ToR8 (IS1<$>$ ToR4<->ES2) to provide connectivity to NFVs. After the provisioning, the controller starts the statistics collection to monitor the packets re-transmitted due to collisions in the switch, and the packets lost at the ToR due to buffer overflow. The OF-agent reads the monitored counters from its controlled FPGA devices and then reports the aggregated per-port values to the ODL controller through the extended OF protocol message as shown in Fig. 3(a). Once the ME detects that the packets losses are over a preventive threshold $9000000(96053079$ > 9000000), it triggers the NS reconfiguration through an alternative path, to maintain the requested QoS. The OPM coordinates with the PCE to calculate the new network connectivity among those ToRs (e.g., ES1<->ToR5<$>$ IS2). Fig.3 (b) shows the new path configuration information at the flow used to update set of actions inside the ToRs and the optical switches controllers. The NS reconfiguration is completed through the new end-to-end connection within $125 \mathrm{~ms}$ which is less than the application switchover time. Once the connections are reconfigured, the packet loss will below the threshold and there will be less retransmitted packets as shown in Fig. $3(\mathrm{c})$.

Second, we assess and validate the dynamic switching operation of the Photonic integrated switch. In particular, we assess the switching operation of the WSS1 of the IS1 module. The switch enabling signals (label control 1-4) generated by the FPGA switch controller after processing the optical labels (RR) of the 4 ToRs, and the channel traces at the WSS1 (output port 1) are reported in Fig. 3(d). The enabling signals are synchronized with the optical packets and a bias current of $40 \mathrm{~mA}$ is applied for the "on" state of the SOA gates. The traces indicate that the optical packets are dynamically switched according to the FPGA control signals. Moreover, the dynamic switch control operation of the packets to the four WSSs output ports has also been validated as shown in Fig. 3(e). Packets from ToR 1 has been switched to the 4 output ports by dynamic controlling the SOA gates of the 4 WSSs. Bit error rate (BER) curves for the switched 4 ToRs channel inputs are reported in Fig. 3(f). The backto-back (B2B) curve is included as reference. Error-free operations with $<1 \mathrm{~dB}$ have been measured for ToR 1 $(\mathrm{CH} 1)$ and ToR 3 (CH 3) packets, while for ToR $2(\mathrm{CH} 2)$ and TOR $4(\mathrm{CH} 4)$ packets the penalty is around $2 \mathrm{~dB}$, but still enough quality to be correctly detected. Those results confirm that the fast dynamic control of the PIS, and validate the switching operation in space, wavelength, and time domain

\section{CONCLUSIONS}

We experimentally assess the SDN enabled reconfigurable optical DCN based on Photonic integrated switches with dynamic NS provisioning and reconfiguration services. Joint efforts of both Controller and Orchestration layers allow for the best optical path layout for NS provisioning, and the network statistics monitoring dynamically triggers the NS reconfiguration within $125 \mathrm{~ms}$ to guarantee the QoS per slice. Experimental results indicate that the Photonic switch based DCN can switch error-free the $10 \mathrm{~Gb} / \mathrm{s}$ traffic in space, wavelength, and time domain with no packets loss and $<2 \mathrm{~dB}$ penalty.

\section{ACKNOWLEDGMENTS}

The authors would like to thank the H2020 Passion (780326) and H2020 Qameleon (780354) projects for partially supporting this work.

\section{REFERENCES}

[1] Cisco Global Cloud Index: Forecast and Methodology, 2016-2021 White Paper, USA, 2018.

[2] Xue, X and Calabretta, N: Flexibility Assessment of the Reconfigurable OPSquare for Virtualized Data Center Networks Under Realistic Traffics, in ECOC, pp. 1-3, 2018.

[3] Xue, $X$ and Calabretta, N: Performance Assessment of OPSquare Data Center Network with Elastic Allocation of WDM Transceivers. In ICTON, pp. 1-4, 2018.

[4] Yan, F and Calabretta, N: HiFOST: A scalable and lowlatency hybrid data center network architecture based on flow-controlled fast optical switches. IEEE/OSA JOCN 10, no. 7 (2018): 1-14.

[5] Calabretta, N and R. Stabile: Photonic integrated WDM cross-connects for optical metro and data center networks. vol. 10946, p. 1094603. International Society for Optics and Photonics, 2019.

[6] Prifti, K., and Stabile, R: System Performance Evaluation of a Nanoseconds Modular Photonic Integrated WDM WSS for Optical Data Center Networks. OFC, pp. Th2A$31,2019$.

[7] Xue, X and F. Wang: Experimental Assessment of SDNenabled Reconfigurable OPSquare Data Center Networks with QoS Guarantees. OFC, pp. M3F-4, 2019. 